\title{
BACTEROIDES INFECTION IN THE SURGERY OF CHILDHOOD*
}

BY

\author{
A. A. GUNN \\ From Western General Hospital, Edinburgh
}

Death from acute appendicitis in childhood is now so rare that its occurrence calls for a retrospective study of any unusual features of the case. A fatal case with complications apparently associated with or the result of infection by the bacteroides group of organisms prompted an investigation into the part played by these bacteria. A review of the literature and one fatal and one surviving case were the subject of a previous paper (Gunn, 1956). Since that time five further cases of bacteroides infection have been encountered. It is the purpose of this paper to record these cases and to emphasize the importance of the early recognition and treatment of this infection. An account is given of the problem as it presented to us.

Case 1. A girl aged 8 years was admitted with a 48-hour history of abdominal pain beginning centrally, spreading to the right iliac fossa and accompanied by nausea and vomiting. The clinical findings were: temperature $101 \cdot 8^{\circ} \mathrm{F}$., pulse rate 120 per minute, tenderness localized to McBurney's point with guarding in the right iliac fossa and rebound tenderness in both iliac fossae, bowel sounds present but faint, white cell count 8,000 per c.mm. A diagnosis of acute appendicitis was made and, at operation two hours later, a gangrenous, unperforated appendix was removed from the pelvis. There was no obvious spread of infection beyond the appendix and the wound was closed without drainage. The appendix contained Gram-negative bacilli and Gram-positive cocci. There was no growth on aerobic culture and anaerobic cultures were not made.

Penicillin and streptomycin were given intramuscularly for the first 48 hours but the general condition did not improve and terramycin was then substituted.

Late on the second post-operative day the child's condition deteriorated rapidly over a few hours. Repeated abdominal examination revealed bruising spreading rapidly around the gridiron incision. The diagnosis of intraperitoneal haemorrhage after slipping of the ligature on the appendicular artery was

* A paper read at the meeting of the British Association of Paediatric Surgeons held in Edinburgh in June, 1957. made and after resuscitation with blood and plasma laparotomy was carried out. When the gridiron incision was opened, the rectus and oblique muscles were black and necrotic with evil-smelling grey pus between the muscle layers. There was no evidence of peritoneal haemorrhage or leakage from the appendix stump. A specimen of pus was taken for investigation and the wound edges were apposed with a few interrupted sutures.

The subsequent course was marked by the development of infective lesions in the superficial tissue, the pleural and pericardial cavities and the subphrenic areas (Figs. 1 and 2). Treatment consisted of aspiration and drainage as indicated, blood transfusion ( 5 pints over 15 days) to counter a falling haemoglobin level and an attempt at antibiotic cover by repeated sensitivity tests, the reports of which were only received after a necessary delay of four days. The organisms isolated, their sensitivity to antibiotics, and the antibiotics administered are detailed in Fig. 3. Penicillin and streptomycin were discontinued because of lack of response and terramycin was substituted. Erythromycin was added because of the passage of loose stools which were sug-

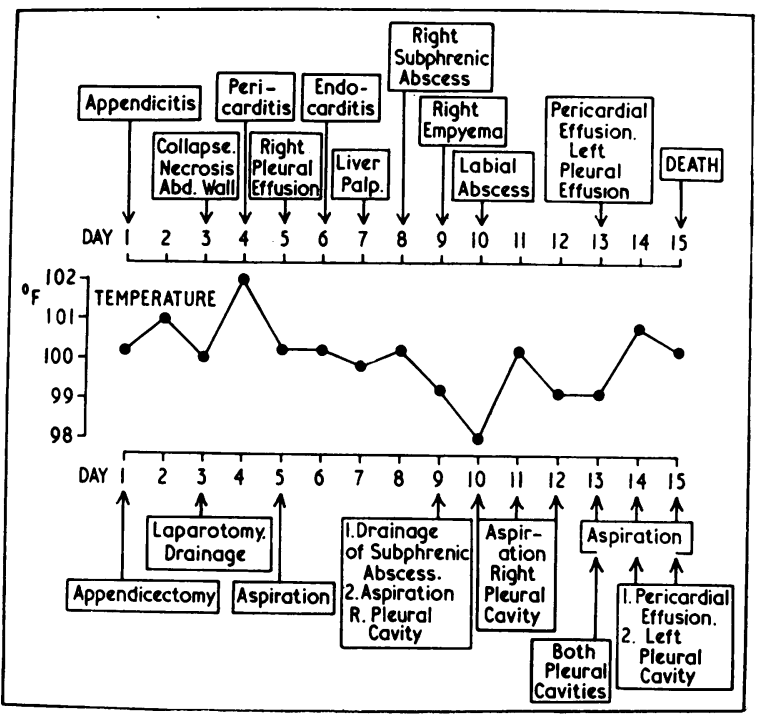

Fig. 1.-Case 1, girl aged 8 years. 


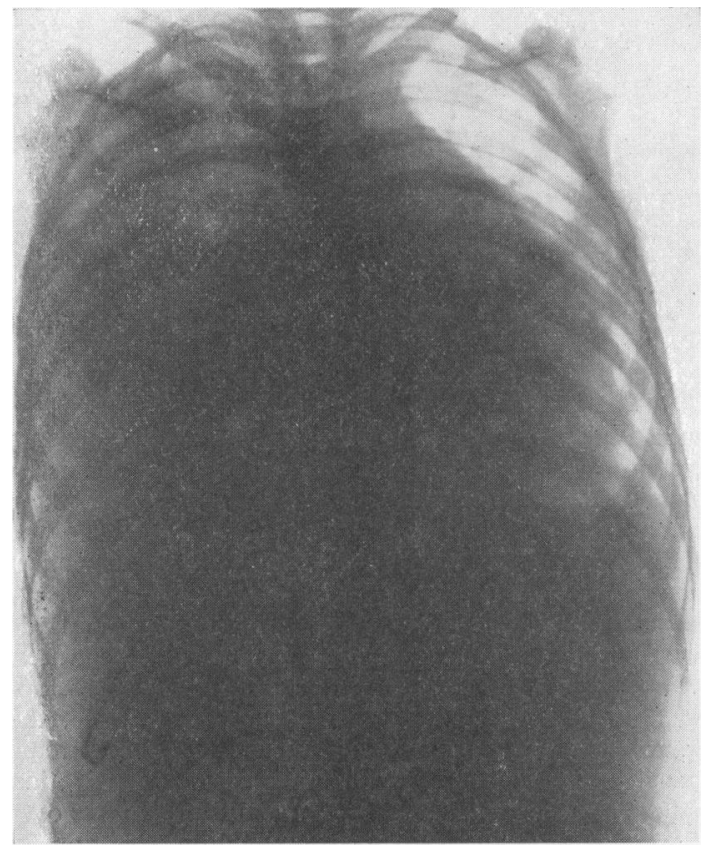

FIo. 2.-Chest radiograph showing pericardial and right pleural effusions.

gestive of staphylococcal enteritis. A further report showed that all the organisms were sensitive to chloramphenicol which was given but the next report suggested that the sensitivity had changed and that penicillin was the drug of choice. This was probably the crucial point as a later report showed that the bacteroides were no longer sensitive to penicillin and from the tenth to fourteenth days the patient received an antibiotic to which the organism was resistant. Death occurred on the fifteenth day.

\section{The Organism}

The bacteroides are a group of Gram-negative, non-sporing, non-motile anaerobic bacilli. They are haemolytic and necrotizing and some are gasproducing. An important characteristic is that these organisms are slow to grow on culture media and may not appear for well over 48 hours. In consequence infection of this variety is often missed, since it is common laboratory practice to discard anaerobic cultures that are negative after 48 hours.

The organisms are of several types and bacteriologists are undecided on their classification. Alston (1955) records the various synonyms and terms infection due to them 'Necrobacillosis'.

For clinical purposes two main groups can be identified.
(1) B. funduliformis, pleomorphic, cocco-bacillary in early infections and later becoming filamentous; these are the more pathogenic variety and are associated with a high morbidity and mortality. (2) B. fragilis are small bacilli of constant form which produce local infections but only rarely septicaemia.

The organisms are normal commensals of the mucous membrane of the mouth, the intestinal and urinary tract and the female genital organs. Under unusual circumstances-generally in a mixed infection with anaerobic streptococci-they may become pathogenic.

Two factors seemed to play a part in the development of pathogenicity: a surgical operation and, as King, Conklin and Collette (1952) suggested, an inherently inferior defence against this type of infection. Thus, when the associated organisms were overcome the bacteroides were still able to proliferate. It is possible, therefore, that bacteroides infection may occur more frequently now that the more usual pathogenic organisms can be effectively controlled by antibiotics.

The recognition of the part played by the bacteroides group of organisms in Case 1 prompted a review of the literature which was the subject of a previous paper and only a brief summary is given here. Records of 330 cases were found and in 148 there was bacteroides septicaemia. There was no difference in the sex incidence, the maximum incidence being from 10 to 40 years although males are

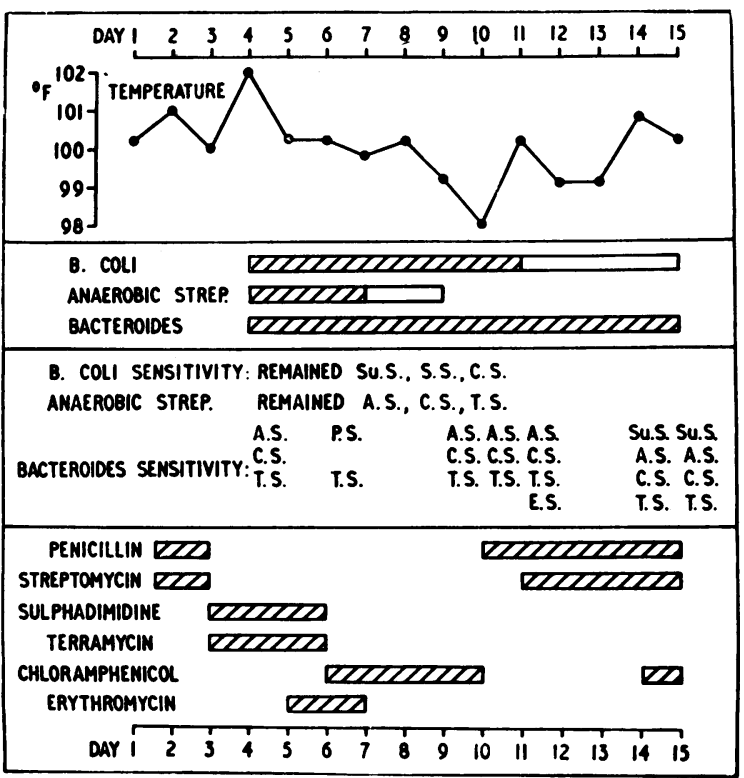

Fig. 3.-Case 1, girl aged 8 years. 
affected at all ages most of the female cases occur between 20 and 30 years.

Bacteroides septicaemia is always serious. Before the introduction of sulphonamides the mortality rate was $80 \%$, with sulphonamides over $50 \%$ and after antibiotics became available the mortality rate remained $33 \%$.

The primary lesions in the septicaemic cases occurred in the nasopharynx, alimentary tract, middle ear, female genital tract and in traumatic wounds. Further reference is made only to the primary lesions which are of importance in childhood.

Nasopharyngeal Infection. This is the most frequently reported source, a common sequence being tonsillitis, tonsillar or peritonsillar abscess, cervical lymphadenitis, cervical abscess and, finally, thrombophlebitis in the internal jugular vein. Frankel (1925) noted that this series was a frequent precursor of septicaemia, and for a time ligation of the internal jugular vein was recommended. Kissling, in 1929, attempted this but 10 of his 12 cases died.

Alimentary Tract. In 26 of the recorded cases septicaemia followed lesions of the alimentary tract. Appendıcitis, pyelophlebitis and liver abscess frequently preceded septicaemia. Such a sequence was not uncommon before antibiotics. Although the organisms could not always be isolated it is possible that bacteroides were, in fact, the offending organisms because the clinical picture resembles so closely that of proven bacteroides septicaemia. serous cavities, liver and lung is most striking although the formidable list of complications is probably incomplete as some of the recorded cases lacked detail.

Clinical Features. The clinical features conform to a regular pattern. (1) Symptoms and signs of the primary lesion; (2) a period of apparent recovery; (3) symptoms and signs of a spreading infection at the site of the original lesion; (4) abrupt onset of septicaemia with rigor, profuse sweating, anaemia and jaundice; (5) symptoms and signs of metastatic infective lesions.

The dramatic picture presented by the first patient and the clarification of the features of the infection from cases recorded in the literature suggested that bacteroides infection was not uncommon and that its occurrence was frequently not recognized. Suspicion of this complication from the clinical features and the routine setting up of anaerobic cultures when suspicion arose has led to the recognition of six further cases (four in children) within a period of 18 months. The case records follow:

Case 2 (Fig. 4). A girl aged 11, with a history of recurrent abdominal pain suggestive of mesenteric adenitis, was admitted in an acute attack. As appendicitis could not be excluded, laparotomy was carried out and the diagnosis of mesenteric adenitis was confirmed. A normal appendix was removed.

A minor post-operative chest infection responded quickly to penicillin and physiotherapy. For two weeks following operation the only complaint was occasional vague abdominal pain for which no cause could be found.
Complications of Bacteroides Infection. The complications of bacteroides infection are detailed in Table 1 . The frequency of abscess formation in the

TABLE 1

COMPLICATIONS OF BACTEROIDES INFECTIONS

\begin{tabular}{|c|c|c|c|c|c|c|}
\hline $\begin{array}{l}\text { Septicaemia } \\
\text { Anaemia } \\
\text { Jaundice } \\
\text { Thrombophlebitis } \\
\text { Abscess in subcuta }\end{array}$ & $\begin{array}{l}\cdots \\
\cdots \\
\cdots \\
\text { aneous }\end{array}$ & $\begin{array}{l}\cdots \\
\cdots \\
\ddot{\text { tissues }}\end{array}$ & $\begin{array}{l}\cdots \\
\cdots \\
\cdots \\
\cdots\end{array}$ & $\begin{array}{l}\cdots \\
\cdots \\
\cdots\end{array}$ & $\begin{array}{l}\cdots \\
\cdots \\
\cdots \\
\cdots\end{array}$ & $\begin{array}{r}173 \\
45 \\
61 \\
26 \\
50\end{array}$ \\
\hline $\begin{array}{l}\text { Peritonitis and int } \\
\text { Liver abscess }\end{array}$ & $\begin{array}{l}\text { ra-abd } \\
\ldots\end{array}$ & lominal & $\begin{array}{l}\text { absc } \\
\ldots\end{array}$ & . & $\begin{array}{l}\cdots \\
\cdots\end{array}$ & $\begin{array}{l}23 \\
53\end{array}$ \\
\hline $\begin{array}{l}\text { Pulmonary absces } \\
\text { Pleural effusion an }\end{array}$ & $\begin{array}{l}\text { s and } \\
\text { nd emp }\end{array}$ & $\begin{array}{l}\text { pneumo } \\
\text { pyema }\end{array}$ & $\begin{array}{c}\text { nia } \\
\cdots\end{array}$ & $\begin{array}{l}\cdots \\
\cdots\end{array}$ & $\begin{array}{l}\cdots \\
\cdots\end{array}$ & 36 \\
\hline $\begin{array}{l}\text { Pericardial effusio } \\
\text { Endocarditis }\end{array}$ & n & $\begin{array}{l}\ldots \\
\cdots\end{array}$ & $\ddot{*}$ & $\cdots$ & 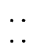 & \\
\hline $\begin{array}{l}\text { Meningitis } \\
\text { Intracranial absces }\end{array}$ & & $\begin{array}{l}\cdots \\
\cdots\end{array}$ & 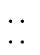 & $\cdots$ & $\ddot{\cdots}$ & \\
\hline $\begin{array}{l}\text { Arthritis } \\
\text { Osteomyelitis } \\
\text { Renal abscess } \\
\text { Prostatic abscess }\end{array}$ & $\begin{array}{l}\cdots \\
\cdots \\
\cdots\end{array}$ & $\begin{array}{l}\cdots \\
\cdots \\
\cdots\end{array}$ & $\begin{array}{l}\cdots \\
\cdots \\
\cdots\end{array}$ & $\begin{array}{l}\cdots \\
\cdots \\
\cdots\end{array}$ & $\begin{array}{l}\cdots \\
\cdots \\
\cdots\end{array}$ & 22 \\
\hline
\end{tabular}

CASE 2

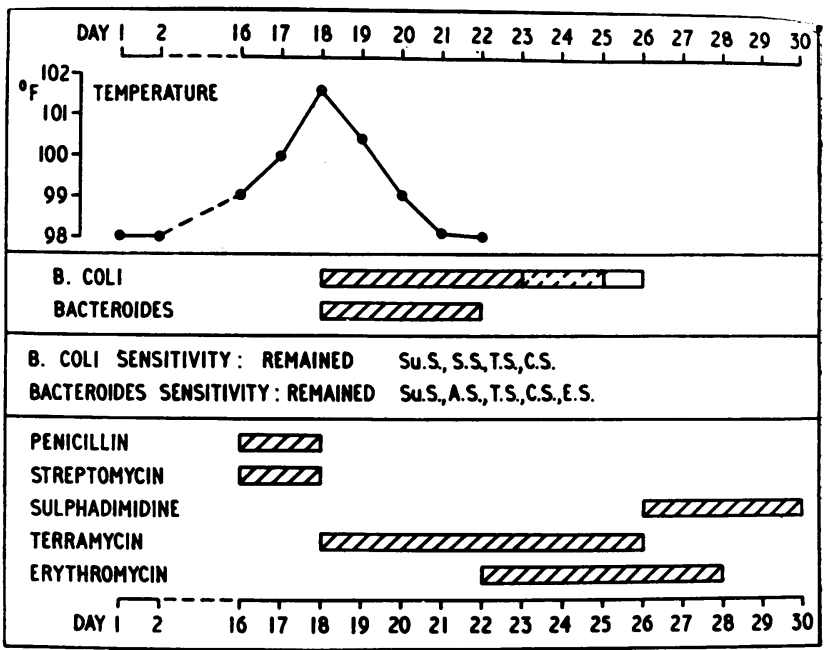

FIG. 4.-Case 2, girl aged 11 years. 
On the fifteenth post-operative day pain became localized to the gridiron incision, where some bruising was apparent. The temperature was $99^{\circ} \mathrm{F}$. but the patient did not appear ill. Penicillin and streptomycin were administered but over the next 48 hours the temperature began to swing and there was profuse sweating. The child became pale and slightly icteric, the abdominal bruising increased and a mass formed in the pelvis and the right iliac fossa.

The possibility of a bacteroides infection was appreciated and terramycin therapy was begun. The gridiron incision was reopened and the muscles of the anterior abdominal wall found to be black and necrotic with collections of evil-smelling pus. As the exposure was not entirely satisfactory a paramedian incision was made and the right rectus muscle was also found to be black and oedematous, even above the level of the umbilicus.

A large, foul-smelling abscess in the pelvis and the right iliac fossa was emptied and drainage established through a suprapubic stab incision. Leakage from the appendix stump could not be demonstrated. The gridiron incision was left open with a hydrogen peroxide pack and the paramedian closed with a few interrupted sutures.

Bacteriological examination confirmed the presence of a bacteroides infection and septicaemia after four days' incubation. Subsequently repeated examinations of the purulent discharge showed no change in the sensitivity to antibiotics.

Terramycin was continued for eight days, and, for the latter half of this course, erythromycin was also given initially to prevent staphylococcal enteritis and later because the organisms were shown to be highly sensitive to it. Therapy was tailed off with sulphadimidine for two weeks.

The temperature fell rapidly but despite transfusion the subsequent progress was slow. On discharge six weeks after admission the wounds had healed but there was still considerable induration in the anterior abdominal wall.

A normal appendix was removed. The case illustrates two important points: (1) the delay in the development of the complication and (2) the administration of an antibiotic before its development.

Case 3 (Fig. 5). A girl aged 11 was admitted with a 12-hour history of colicky abdominal pain, later becoming constant. There was associated nausea and vomiting. She had had two previous attacks of pain.

On examination, there was tenderness in both iliac fossae, maximal on the right side. There was no rebound tenderness but she was very tender on rectal examination.

A diagnosis of appendicitis was made and at operation an acutely inflamed appendix was removed and the wound closed without drainage.

Two days later the temperature began to swing and was considered to be due to wound infection. Penicillin and streptomycin were administered.

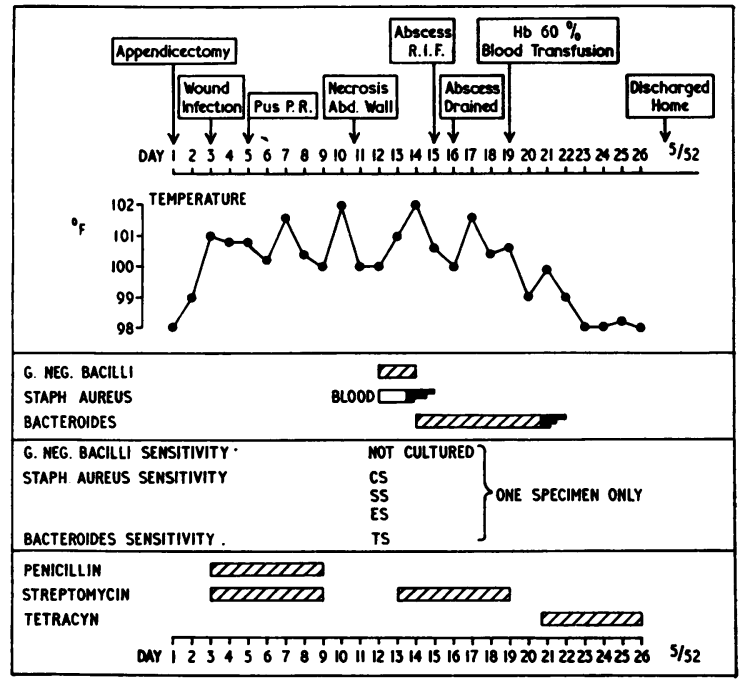

FIG. 5.-Case 3, girl aged 11 years.

Five days post-operatively the patient developed diarrhoea and was found to be very tender in the pouch of Douglas. Twelve days post-operatively bruising became apparent around the wound and probing liberated about $60 \mathrm{ml}$. of dark blood which, on culture, proved to contain bacteroides. Fourteen days postoperatively it was decided to drain the abscess which was found to extend into the pelvis. Six hundred millilitres of pus were liberated. A drain was left down into the pelvis. In addition, tetracycline was given.

The subsequent progress was steady and the girl was discharged five weeks after admission.

This was a case of acute appendicitis, presenting again (1) the delay in the full development, (2) the good response to adequate drainage, (3) the failure to respond to penicillin and streptomycin but a response to tetracycline.

Case 4 (Fig. 6). A man aged 30 was admitted with a 12-hour history of pain moving from the right hypochondrium to the right iliac fossa with nausea and vomiting. There was one previous attack.

Examination revealed tenderness and rigidity in the right iliac fossa. A diagnosis was made of acute appendicitis and at operation a pelvic, gangrenous appendix was removed and the wound closed with drainage of the peritoneal cavity. Penicillin was administered.

Post-operatively the drain discharged evil-smelling grey pus and bacteriological examination revealed a mixed growth of $\beta$ haemolytic streptococci and bacteroides. The latter was insensitive to penicillin.

The temperature began to swing despite apparent adequate drainage and a pelvic abscess was found. Terramycin was given and the patient recovered without surgical or spontaneous drainage of the abscess. 
This was a case of gangrenous appendicitis presenting (1) abscess collections despite drainage;

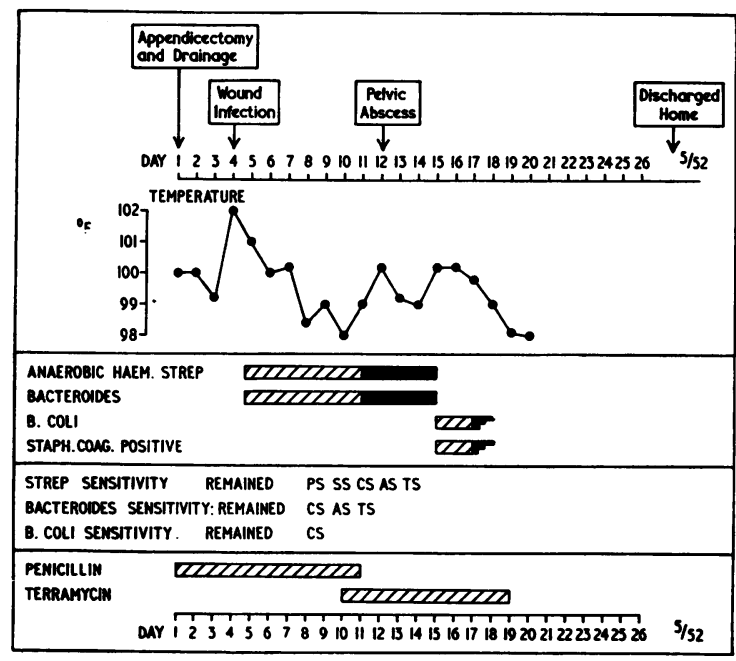

Fig. 6.-Case 4 , man aged 30 years.

(2) once again penicillin therapy, probably administered incorrectly for an alimentary infection; (3) the delay in appearance of collections.

Case 5 (Fig. 7). A boy aged 3 was admitted with a history of a cold and cough for one week and pain in the right iliac fossa for 24 hours. In addition he had nausea and vomited.

On examination there was tenderness in the right iliac fossa with guarding over the whole lower abdomen.

A diagnosis of acute appendicitis was made and at operation a perforated appendix was removed from a retrocaecal extraperitoneal position. The wound was not drained.

Three days later he began to vomit and the temperature began to swing. The wound showed bruising. He looked toxic, the possibility of a bacteroides infection was considered, and drip and suction and terramycin therapy were instituted.

Eight days after operation he developed a superficial and deep venous thrombosis in the right leg. Heparin was administered for five days.

On the eleventh day erythromycin was given instead of terramycin because of the appearance of an abscess of the anterior abdominal wall.

On the 15th day the abdominal abscess discharged spontaneously with a copious flow of brownish, foulsmelling pus. Four weeks after operation the wound had almost healed and the oedema of the right leg had subsided. Eight weeks after appendicectomy he was discharged home.

This case of perforated appendicitis illustrated two factors: (1) local spreading infections after a delay of some two weeks, (2) evidence of thrombophlebitis.

Case 6 (Fig. 8). A man aged 32 was admitted with two weeks' history of suprapubic pain spreading to the right iliac fossa. He had felt sick but had not vomited. Two days before admission the pain became more severe and was located just to the right of the umbilicus.

On examination his temperature was $101^{\circ} \mathrm{F}$. and a tender swelling was palpated in the right iliac fossa with overlying guarding. A diagnosis of appendicitis with abscess formation was made and laparotomy was carried out. The mass was found to be a large abscess within the mesentery of the mid-ileum surrounded by coils of small bowel. The adjacent ileum was thickened and rigid. A biopsy of the abscess cavity wall was taken. The bacteriological report showed bacteroides.

His immediate post-operative progress was very stormy with a peritonitis which was treated by drip and suction and penicillin and streptomycin.

On the fifth post-operative day terramycin was substituted because of the patient's failure to respond.

On the seventh post-operative day the patient became severely shocked very suddenly. This was countered with a massive intravenous transfusion of seven parts plasma, and one of blood. In the same evening the patient developed profuse diarrhoea. Later bacterio-

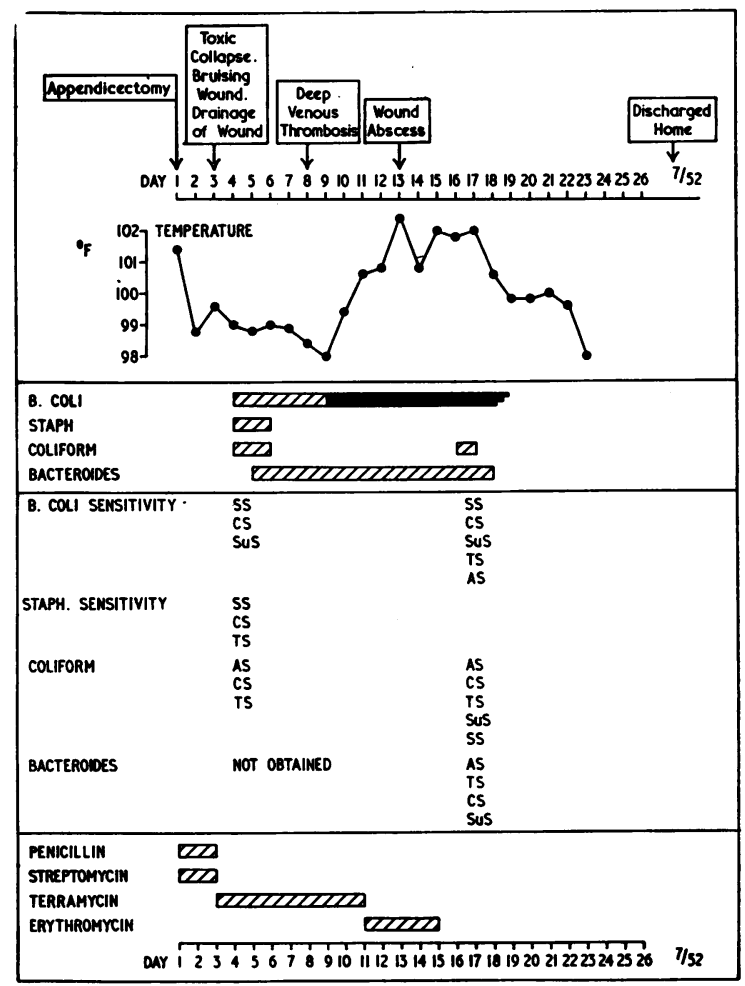

Fig. 7.-Case 5, boy aged 3 years. 
logical reports showed this to be a staphylococcal enteritis; the staphylococci were erythromycin-resistant.

On the eighteenth post-operative day, when the patient had begun to improve, he started complaining of abdominal pain and the temperature began to swing. A wound abscess was obviously gathering and required

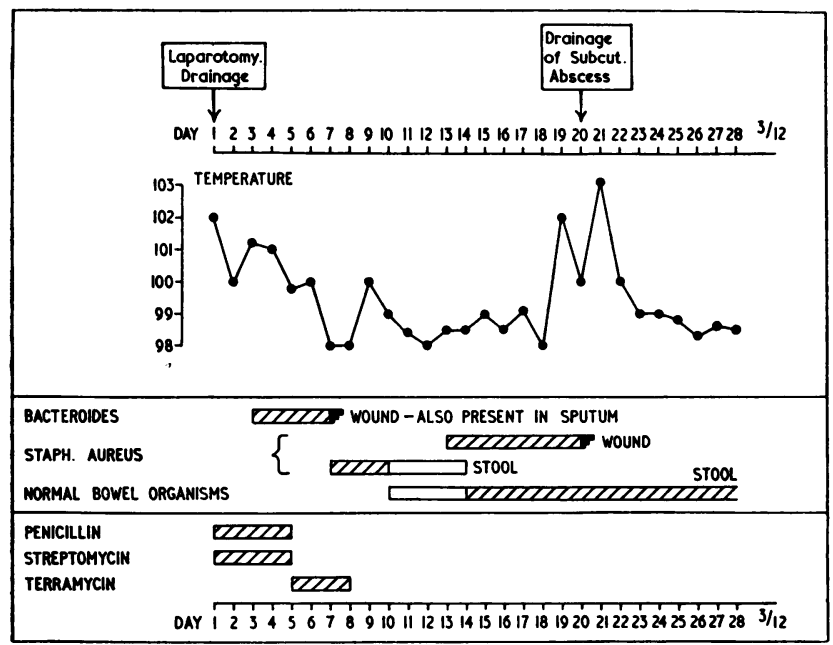

Fig. 8.-Case 6, man aged 32 years.

operative drainage on the twentieth day. The wound was left open and was healed one month later.

This case, previously reported by Dudley and MacLaren (1956), is interesting from many points of view. The points requiring emphasis are: (1) A bacteroides bowel infection present from the onset; (2) later development of staphylococcal enteritis; (3) final development of a wound infection on the 18th day which responded well to open drainage.

Case 7 (Fig. 9). A boy aged 4 was admitted with a two weeks' history of tonsillitis for which he had received penicillin from his own doctor. Despite this a mass had developed in the right side of the neck.

On examination the temperature was $98.8^{\circ} \mathrm{F}$., the fauces were inflamed. On palpation a large fluctuant swelling was found in the right posterior triangle, 12.5 $\mathrm{cm} . \times 7 \mathrm{~cm}$. The swelling extended from the postauricular region, anteriorly, to the angle of the mandible and, posteriorly, to the occiput.

After adequate drainage of the abscess his condition gradually improved until he was discharged on his sixth week in hospital.

The sequence was tonsillitis followed by lymphadenitis and abscess. This case is of interest for three reasons: (1) It is the type of case most commonly reported; (2) failure to respond to penicillin; (3) anaemia, haemoglobin falling to $70 \%$.

\section{Conclusion}

Diagnosis. The diagnosis of bacteroides infection and septicaemia is not difficult if its possible occurrence is kept in mind. Confirmation is obtained by careful bacteriological studies. The initial film and prolonged anaerobic culture will reveal the organisms.

Treatment. The principles of treatment can be summarized briefly as follows: (1) Open treatment of infected wounds; (2) prompt surgical drainage of any purulent collection; (3) the exhibition of a broadspectrum antibiotic, usually more effective than penicillin or streptomycin; (4) continuous bacteriological control, since sensitivity to antibiotics may change with great rapidity; (5) maintenance of haemoglobin and of general nutrition.

\section{Prognosis}

Bacteroides infection has been attended hitherto by a high mortality and serious morbidity. This is well-illustrated by the cases presented, five of the seven being under the age of 12 years.

The mortality and morbidity can be reduced only when the diagnosis is made quickly and appropriate treatment is started in good time. Bacteriological confirmation may take as long as four days, and, if the infection is suspected on clinical grounds, treatment should be begun immediately without awaiting the bacteriological report.

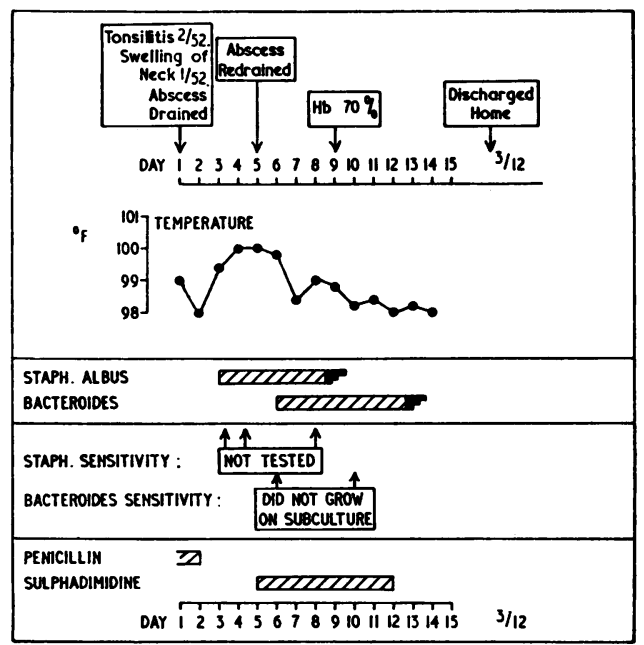

Fig. 9.-Case 7, boy aged 4 years. 
From the recorded cases and the experience gained from the treatment of the seven patients whose histories are described it seems justifiable to conclude that surgeons and bacteriologists are insufficiently aware of the occurrence of anaerobic infection by this group of organisms and the late development of septicaemia. Since so much can be gained from early treatment it is imperative that anaerobic cultures are not discarded after 48 hours' incubation when the disease is suspected on clinical grounds.
I am grateful to Mr. J. J. Mason Brown, Mr. F. H. Robarts, Mr. I. S. Kirkland, Mr. H. A. F. Dudley and Mr. Ian Campbell for asking me to see and treat patients under their charge.

\section{REFERENCES}

Alston, J. M. (1955). Brit. med. J., 2, 1524.

Dudley, H. A. F. and MacLaren, I. F. (1956). Lancet, 2, 1182.

Frankel, E. (1925), Cited by Kissling, K. (1929). Münch. med. Wschr., 76, 1163.

Gunn, A. A. (1956). J. roy. Coll. Surg. Edinb. 2, 41

King, A. B., Conklin, S. D. and Collette, T. S. (1952). Ann. intern.

Med., 37, 761.
Kissling, K. (1929). Münch. med. Wschr., 76, 1163. 